\title{
THE SIGNATURE OF SMOOTHINGS OF HIGHER DIMENSIONAL SINGULARITIES
}

\author{
BY STEPHEN SHING-TOUNG YAU ${ }^{1}$ \\ Communicated by Hyman Bass, April 28, 1977
}

1. Introduction. Let $f:\left(C^{2 n+1}, 0\right) \rightarrow(C, 0)$ be the germ of a complex analytic function with an isolated critical point at the origin. For $\epsilon>0$ suitably small and $\delta$ yet smaller, the space $V^{\prime}=f^{-1}(\delta) \cap D_{\epsilon}$ (where $D_{\epsilon}$ denotes the closed disk of radius $\epsilon$ about 0 ) is a real oriented $2 n$-manifold with boundary whose diffeomorphism type depends only on $f$. It has been proved by Milnor [5] that $V^{\prime}$ has the homotopy type of a wedge of $2 n$-spheres, and the number $\mu$ (Milnor number) of $2 n$-spheres is readily computable. Recently an interesting formula for $\mu$ was given in terms of analytic invariants of a resolution of the singularity at 0 of $f^{-1}(0)$. For surface singularities this is due to Laufer [4]. For higher dimensional singularities this is due to Bennett and the author [1]. In case of two dimensional singularities, various signature formulae are known. For example, Hirzebruch and Mayer [3] have a formula for the signature when $f$ is of the type $x^{a}+y^{b}+z^{c}$ and Steinbrink [6] has a formula for the signature when $f(x, y, z)$ is weighted homogeneous. Recently Durfee [2] has given a formula for the signature $\sigma$ of $V^{\prime}$ in terms of topological invariants of a resolution of the singularity at 0 of the complex surface $f^{-1}(0)$. In this paper a formula for the signature of even dimensional singularities is given in terms of topological invariants and analytic invariants of a resolution of the singularity. As a consequence of this formula, a duality theorem for even dimensional strongly pseudo. convex manifolds is proved. It is a pleasure to acknowledge the constant encouragement of Professor Henry Laufer in this research. We would also like to thank Professor Bennett, Professor Coleff, Professor Deligne and Professor Siu for their helpful discussion of mathematics.

2. Main results. The ingredient of the proof of Laufer's formula [4] is the Riemann-Roch Theorem. The ingredient of the proof of Durfee's formula (Theorem 1.5 of [2]) is the Hirzebruch index Theorem. We prove the following theorem without using the Riemann-Roch Theorem or the Hirzebruch index Theorem.

THEOREM 1. Let $f(x, y, z)$ be holomorphic in $N$, a Stein neighborhood of

AMS (MOS) subject classifications (1970). Primary 13C40, 13C45, 14B05, 14E15, 32C40; Secondary 14J15, 14C20.

${ }^{1}$ Supported in part by NSF grant MCS72-05055 A04. 
$(0,0,0)$ with $f(0,0,0)=0$. Let $V=\{(x, y, z) \in N: f(x, y, z)=0\}$ have $(0$, $0,0)$ as its only singular point. Let $\mu$ be the Milnor number of $(0,0,0)$ and $\sigma$ be the signature of $V^{\prime}$. Let $\pi: M \rightarrow V$ be a resolution of $V$ and $A=$ $\pi^{-1}(0,0,0)$. Let $\chi_{T}(A)$ be the topological Euler characteristic of $A$ and $s$ be the number of irreducible components of $A$. Then

$$
1+\mu+\sigma=4 \operatorname{dim} H^{1}(M, 0)-s+\chi_{T}(A) .
$$

If we combine Theorem 1 and Durfee's formula for signature (Theorem 1.5 of [2]), we obtain Laufer's formula for $\mu$ in [4]. Since the proof of Durfee's formula does not depend on the Riemann-Roch Theorem, we derive Laufer's formula without using the Riemann-Roch Theorem. The following upper estimate of $\sigma$ is obtained, which was not known before.

TheOREM 2. Let the notations be as above. Let $\Omega^{1}$ be the sheaf of germs of holomorphic 1-forms on $M$. Then

$$
\sigma \leqslant-s+2 \operatorname{dim} H^{1}(M, 0)+\operatorname{dim} H^{1}\left(M, \Omega^{1}\right) .
$$

The proof of Durfee's formula for signature (Theorem 1.5 of [2]) is topological. He made use of the concept of signature defect, which is not needed in the proof of the following theorem.

THEOREM 3. Let the notations be as above. Let $K$ be the canonical divisor on $M$. Then

$$
\sigma=-K^{2}-s-8 \operatorname{dim} H^{1}(M, 0)
$$

By combining formulas (1) and (3), we obtain Durfee's formula for signature. Therefore Durfee's method of signature defect can be completely avoided. If one looks at Durfee's proof of signature formula more closely, one observes that he did not make use of the complex structure of the singularity. By taking account of the complex structure of the singularity, we obtain signature formula for arbitrarily even dimensional singularities.

Definition 4. Let $\pi: M \rightarrow V=\left\{x \in \mathrm{C}^{2 n+1}: f(x)=0\right\}$ be a resolution of the singularity. The signature of $M$, denoted by $\sigma(M)$, is defined to be the signature of $\pi^{-1}\left(D_{\epsilon} \cap V\right)$ where $D_{\epsilon}$ denotes the closed ball of radius $\epsilon$ about 0 , i.e. the signature of a closed tubular neighborhood of the exceptional set $A=$ $\pi^{-1}(0)$.

THEOREM 5. Let $f\left(z_{0}, z_{1}, \ldots, z_{2 n}\right)$ be holomorphic in $N \subseteq \mathrm{C}^{2 n+1}$, $\boldsymbol{n}>1$, a Stein neighborhood of $(0,0, \ldots, 0)$ with $f(0,0, \ldots, 0)=0$. Let $V=\left\{\left(z_{0}, z_{1}, z_{2 n}\right) \in N: f\left(z_{0}, z_{1}, \ldots, z_{2 n}\right)=0\right\}$ have $(0,0, \ldots, 0)$ as its only singular point. Let $\sigma$ be the signature of $V^{\prime}$. Let $\pi: M \rightarrow V$ be a resolution of $V$. Let $\Omega^{p}$ be the sheaf of germs of holomorphic p-forms on $M$. Let $\chi^{p}(M)=\Sigma_{i=1}^{2 n}(-1)^{i} \operatorname{dim} H^{i}\left(M, \Omega^{p}\right)$. Then 


$$
\sigma=-\sum_{p=q}^{2 n-q} \chi^{p}(M)-2 \sum_{p=0}^{q-1} \chi^{p}(M)+\sigma(M)
$$

for $2 \leqslant q \leqslant n$. In case $n=1$, the analogous statement is Theorem 2 because $\sigma(M)=-s$ in this case.

THEOREM 6. Let $M$ be a strongly pseudoconvex manifold of dimension $2 n$. Suppose the maximal compact analytic subset in $M$ can be blown down to isolated hypersurface singularities. Let $\Omega^{p}$ be the sheaf of germs of holomorphic $p$ forms on $M$. Let $\chi^{p}(M)=\Sigma_{i=1}^{2 n}(-1)^{i} \operatorname{dim} H^{i}\left(M, \Omega^{p}\right)$. Then

$$
\chi^{p}(M)=\chi^{2 n-p}(M), \quad 2 \leqslant p \leqslant n .
$$

\section{REFERENCES}

1. B. Bennett and S. S.-T. Yau, Some global formula for Milnor number (submitted).

2. A. Durfee, The signature of smoothings of complex surface singularities (preprint).

3. F. Hirzebruch and $\mathrm{K}$. Mayer, $O(n)$-Mannigfaltigkeiten, exotische Sphären und Singularitäten, Lecture Notes in Math., vol. 57, Springer-Verlag, New York, 1968.

4. H. Laufer, On $\mu$ for surface signularities, Proc. Sympos. Pure Math. 30 (1976), $45-49$.

5. J. Milnor, Singular points of complex hypersurfaces, Ann. of Math. Studies, no. 61 Princeton Univ. Press, Princeton, N. J.; Univ. of Tokyo, Tokyo, 1968.

6. J. Steinbrink, Intersection form for quasi-homogeneous singularities, University of Amsterdam report 75-09, 1975.

DEPARTMENT OF MATHEMATICS, HARVARD UNIVERSITY, CAMBRIDGE, MASSACHUSSETTS 02138 